\title{
OverSoc: Social Profile Based Overlays
}

\author{
David Isaac Wolinsky, Pierre St. Juste, P. Oscar Boykin, Renato Figueiredo \\ University of Florida
}

\begin{abstract}
Online social networking has quickly become one of the most common Internet activities. As social networks evolve, they encourage users to share more information, requiring the users, in turn, to place more trust into social networks. In centralized systems, this means trusting a third-party commercial entity, like Facebook or MySpace. Peer-to-peer (P2P) systems can enable the creation of online social networks extending trust to friends only. In this paper, we present a novel approach to constructing completely decentralized social networks through P2P overlays, OverSoc. Our approach relies on a common directory overlay, which facilitates friend discovery and bootstraps connectivity to individualized profile overlays. Each user has their own individual profile overlay managed transparently using a public key infrastructure (PKI). We define necessary interfaces for constructing the system and describe some examples of user interactions with the system.
\end{abstract}

\section{INTRODUCTION}

Online social networking has become pervasive in daily life, though as social networks grow so does the wealth of personal information that they store. Once information has been released on a social network, known as a user's profile, the user and the data are at the mercy of the terms dictated by the social network infrastructure, which today is typically third-party, centrally owned. If the social network engages in activities disagreeable to the user, due to change of terms or opt-out programs not well understood by users such as recent issues with Facebook's Beacon program [1], the options presented to the user are limited. The options include leaving the social network, surrendering their identity and features provided by the social network; accepting the disagreeable activities; or to petition and hope that the social network changes its behavior.

As the use of social networking expands to become the primary way in which users communicate and express their identity amongst their peers, the users become more dependent on the policies of social network infrastructure owners. Recent work [2] explores the coupling between social networks and $\mathrm{P} 2 \mathrm{P}$ systems as a means to return ownership to the users, noting that a social network made up of social links is inherently a P2P system with the aside that they are currently developed on top of centralized systems. In this paper, we extend this idea with focus on the topic of topology; that is, how to organize social profiles that leverage the benefits offered by a structured P2P overlay abstraction.

Structured P2P overlays provide a scalable, resilient, autonomic platform for distributed applications. Structured overlays enable users to easily create their own decentralized systems for the purpose of data sharing, interactive activities, and other networking-enabled activities. In this paper, we extend our previous work [3], [?] to enable social network profile overlays. The previous work addresses the challenges of bootstrapping secure, private overlays in environments constrained by network address translators (NATs) and firewalls through a public overlay used for discovery and as a relay or communication transport.

A typical social network consists of users and groups. Each user has a profile, a set of friends, and the ability to send and receive private messages; each group consists of one or more managers, users, and a messaging board. Profiles contain user's personal information, status updates, and public conversations, similar to a message board. Friends are individuals trusted sufficiently by a user to view the user's profile. Private messaging sends messages discretely between users without leaking the message to other members. Groups have similar features, though identity is shared by many users.

Using this social networking model, we have designed OverSoc. OverSoc uses a public overlay as a directory for finding and befriending peers or finding and accessing groups. Once group and profile access has been offered, the public overlay can be used to bootstrap connectivity to existing profile and group overlays. Security for a profile is provided by a public key infrastructure (PKI), where profile owners or group managers are the certificate authorities (CA) and all members have signed certificates. The overlay stores profile data or group information in its distributed data store, supporting decentralized access using scalable mechanisms regardless of the profile owner's online presence. In this paper, we present the architecture of these overlays, as presented in Figure 1.

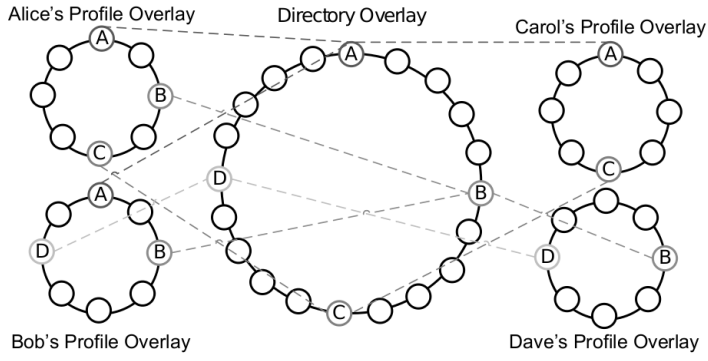

Fig. 1. An example OverSoc social overlay network. Alice has a friendship with Bob and Carol, hence both are members of her profile overlay. Bob has a friendship with Alice and Dave but not Carol; hence Alice and Dave are members of his profile overlay, while Carol is not. Each peer has many overlay memberships but a single root represented by dashed lines in various shades of gray. For clarity, overlay shortcut connections are not shown.

The rest of this paper is organized as follows. Section II provides background and related work. Section III describes OverSoc, explaining how to map social networks onto structured P2P overlays. We express our expectations for user interaction with the system in Section IV. In Section V, we explore some of the remaining challenges introduced by our approach. We conclude the paper in Section VI. 


\section{BACKGROUND}

In this section, we review structured P2P overlays, challenges and solutions for bootstrapping overlays, and other methods for constructing decentralized online social networks. P2P systems build upon existing decentralized concepts by creating scalable, autonomic environments, thereby limiting user exposure to the gory details of system configuration and organization. Since our system consists of many profile overlays, we focus on the challenges of bootstrapping P2P systems, when the system lacks dedicated bootstrapping nodes.

\section{A. Structured P2P Overlays}

P2P systems typically come in two flavors: unstructured and structured. Unstructured systems [4], [5] are generally constructed by peers attempting to maintain a certain amount of connections to other peers in the P2P system, whereas structured systems organize into well-defined topologies, such as trees, 1-D rings, or hypercubes. Though unstructured systems are typically simpler to bootstrap and maintain, they rely on global knowledge, flooding, or stochastic techniques to search for information in an overlay, creating potential scalability constraints. Alternatively, structured systems [6], [7], [8], [9], [10] have guaranteed search time typically with a lower bound of $O(\log N)$ and in some cases even $O(1)$ [11]. The most common feature found in structured overlays is the support for a decentralized storage / retrieval system called a distributed hash table (DHT), that maps keys with associated data to specific node IDs in an overlay.

The bootstrap problem presents a challenge to scenarios discussed in this paper, where peers will be joining many overlays with overlay peers behind NATs. Our previous work [12] presents an abstraction layer to bootstrapping across existing overlays. The key components are a method for reflection in order to obtain publicly reachable addresses, so peers behind network address translators and firewalls can receive incoming connection requests; communication relaying to share public addresses and communicate when direct communication is not feasible; and rendezvous for discovering remote peers, when the overlay lacks stable membership. The solution has been applied to structured overlays, that enabling peers to bootstrap structured overlays from a structured overlay with nodes constrained by NATs. In this application, a peer obtains a public address mapping through reflection using the public overlay. Upon connection, the peer has has the ability to route packets through recursive routing on the overlay, and uses the DHT to discover other peers in the private overlay. Peers multiplex the same UDP and TCP sockets in the private overlay to reuse the reflection services provided in the public overlay.

\section{B. Peer-to-Peer Social Networks}

In [13], Buchegger et al. describe how to use a DHT to store social networking profile. The DHT provides look-up services for storing meta-data pertaining to a peer's profile. Peers query the DHT for updated content from their friends by hashing their unique identifiers (e.g. friends' email addresses). The retrieved meta-data contains information for obtaining the profile data such as IP address and file version. Their work relies on a PKI system that provides identification, encryption, and access control. In contrast, OverSoc maps individual user profiles and groups to a private overlay secured by point-topoint encryption and authentication amongst all peers in the overlay. The private overlay provides a clean abstraction of access control, whereby once admitted to a private overlay, users can access a distributed data store which holds the contents of the owner's profile.

Shakimov et al. in [14] take a different approach by depending on virtual individual servers (VIS) hosted on a cloud infrastructure such as Amazon EC2. Friends contact each other's VIS directly for updates. A DHT is used as a directory for groups and interest-based searches. Their approach assumes bidirectional end-to-end connectivity between each VIS, where a profile is only available during the up time of the VIS. Because of the demands on network connectivity and up time, the approach assumes a cloud-hosted VIS and has difficulty being used on user-owned resources. OverSoc allows peers to have asymmetric connectivity and does not require constant up time through the use of NAT traversal support and the ability to store the profile in the overlay's distributed data store.

The approach presented by Cutillo et al. in [15] relies on a central system to host identities and certificates that can then be used to query a DHT to discover an initial hop in a route to a specific peer through their circle of friends. The circle of friends consists of an unstructured overlay, where direct friends maintain direct connections with the peer, and outer circles consist of friends of friends and friends of friends of friends. The main goal of this work is to remove the private components of a profile from a central entity, whereas OverSoc makes a clean break from all centralization and enables scalability through distributed replication techniques.

Unlike the above approaches, the P2P social network presented by Abbas et al. in [16] uses an unstructured overlay without a DHT where peers connect directly to each other rather than through the overlay establishing unique identifiers to deal with dynamic IPs. Peers cache each other's data to improve availability, while helper nodes are used to assist with communication between peers behind NATs. The approach lacks security and access control considerations and lacks the guarantees and the simplicity of the abstraction offered by a structured overlay.

\section{Social OVERLAYS}

In this section, we explain how OverSoc maps online social networking to virtual private overlays consisting of a public directory overlay with many private profile overlays. The directory overlay supports friend discovery and verification and stores a lists of peers currently active in each profile overlay. Profile overlays support message boards, private messages, and media sharing.

\section{A. Finding Friends}

In a traditional social network, directories are used to search for users based upon public information, such as the user's full 


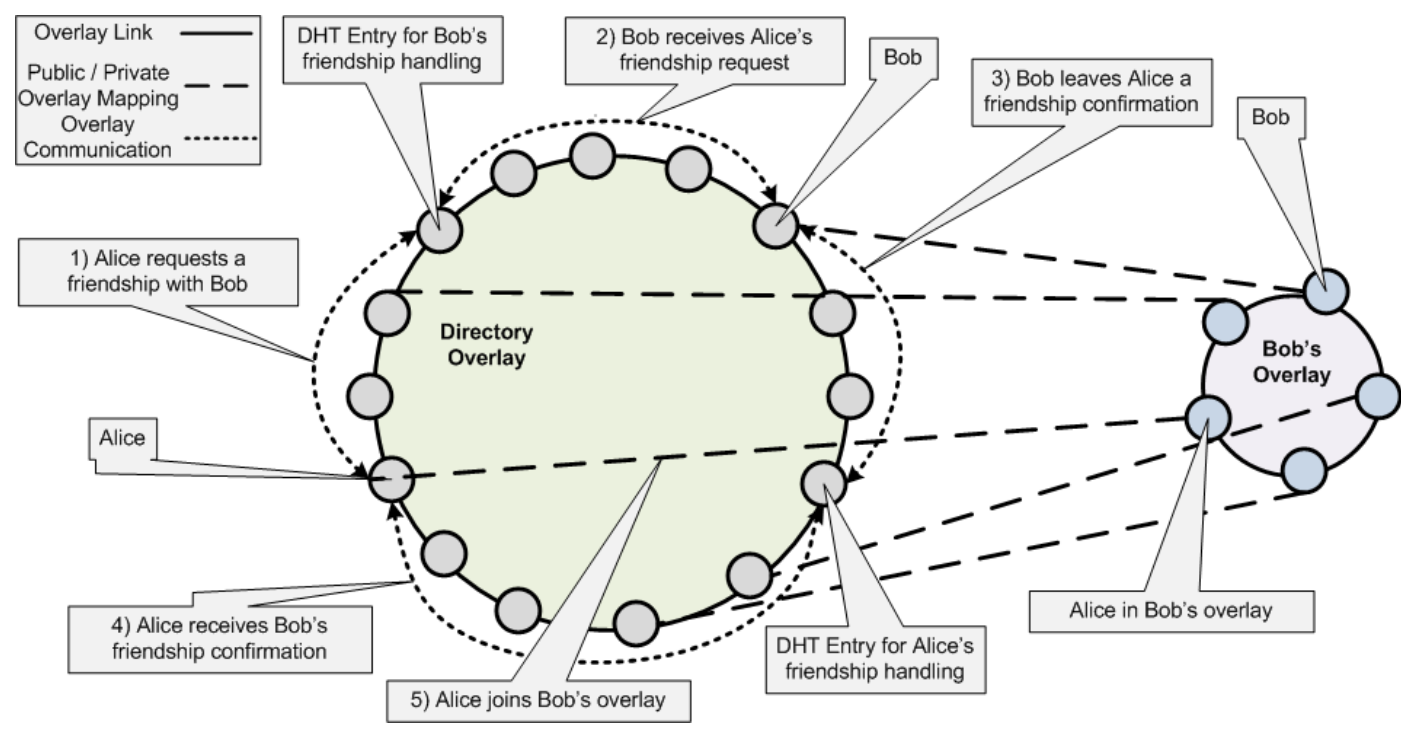

Fig. 2. Alice requests and receives a friendship from Bob. name, user ID, e-mail address, group affiliations, and friends. The resulting search returns zero or more matching directory entries. In OverSoc, directory entries are inserted into the DHT of a public overlay. Since the public information has many components, various subsets form DHT keys that all point to a common, complete listing of the matching public information. For example, a user can store a pointer at the DHT key hash("alice") or hash ("alicebob"). The key here is that any subset of the user's public information in lowercase format can be hashed into a DHT index that would eventually direct the searching user to one or more users' public information. More explicit searches could sift through the results and present to the user only those peers matching all the search parameters. The amount of information shared publicly should be configurable by the user.

While looking for an individual, a peer may discover that many individuals have overlapping public information components, such as the user's name. Assuming all entries are legitimate, the overlay must have some method of supporting multiple, distinct values at the same key, requiring the application and user to parse the responses and determine the best match by reviewing the contents of each certificate. Alternatively, a technique like Sword [17], which supports attribute based searching, could be used to efficiently find peers in an overlay.

To address trust levels when searching for friends, a PGP certificate can be used to store user's public information and verify user's friends and groups. In OverSoc, the main portion of a PGP certificate contains information such as user name, full name, e-mail address, potentially other userdefined data, and signature packets from the user and those that trust the certificate including groups and individuals. These signature packets represent a list of verifiable friends and groups assisting to further uniquely identify a user. Each time a user befriends someone, they should exchange signature packets containing at a minimum the friend's PGP certificate ID, a signature expiration time, and a signature binding this information with the new friend's existing PGP certificate.

This increases the trust level of individuals searching for others especially if they have common friendships or group membership. The use of a time stamp in the signature assists in deciding whether or not a friendship link is still active without accessing the profile overlay of either peers. Thus peers that maintain friendships need to periodically exchange signature packets.

\section{B. Making Friends}

In this example, Alice becomes friends with Bob, as illustrated in Figure 2. Once a user, Alice, has found a friend candidate, Bob, Alice can issue a friendship request and store it in the DHT using the hash of Bob's certificate as an index, this acts a public overlay mailbox. Bob can review the public information of Alice prior to making a decision. If Bob accepts the request, Alice and Bob exchange signature packets and are granted access to each other's profiles. Once profile access has been enabled, the Alice and Bob can learn more information, and if it turns out to be a mistake, either one of them can unilaterally end the relationship.

Alice's friendship request should contain a pointer to her certificate in the overlay, a time stamp, and Bob's certificate identifier. The friendship request is encrypted using Bob's public key and signed using Alice's private key for the purposes of anonymity and authenticity. When Bob receives the friendship request, he can verify that the request was made for Bob by Alice. Upon receiving the friendship request, he has three choices: a conditional accept, an unconditional accept, or a reject. During an unconditional accept, Bob signs Alice's PGP certificate and issues a request to befriend her. Alternatively, he could issue a request to befriend her and wait for her to sign his certificate and investigates her profile prior to signing hers.

Discovery of a user is not limited to the directory entries. Because users have a public overlay based mailbox, they are not required to discover each other only through the directory. Instead, they can use out of band discovery, using mechanisms like e-mail, chat, or personal websites to exchange certificates. 


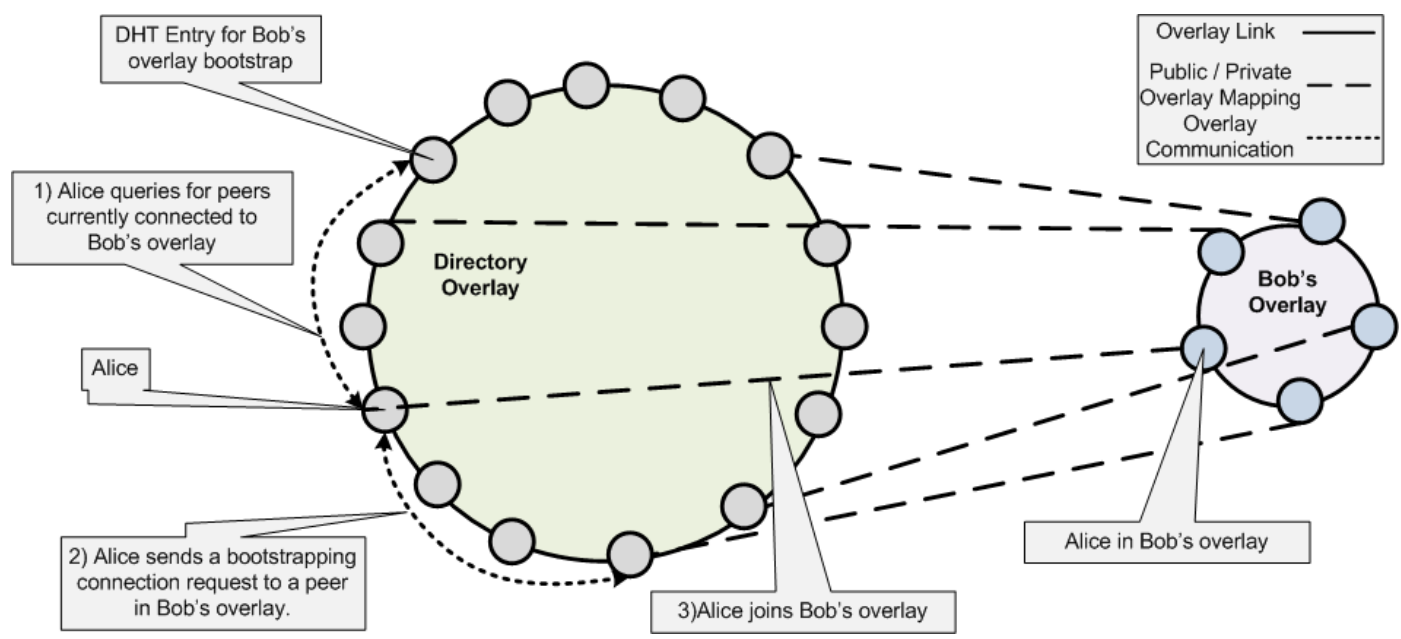

Fig. 3. Alice, already a friend of Bob, connects to his social overlay.

Once a peer has received another peer's certificate, they can submit secure friendship requests using the public overlay. In fact, this sort of system can leverage the trust established by an existing social network to sign and exchange OverSoc's certificates.

\section{The Profile Overlay}

In a traditional social network, the profile or user-centric portion consists of private messaging, data sharing, friendship maintenance, and a public message board for status updates or public messages. In this section, we explain how these components can be applied to a structured overlay dedicated to an individual profile.

Using the techniques such as those described in [3], it is feasible to efficiently multiplex a P2P system across multiple, virtual private overlays enabling each profile owner to have a profile overlay consisting of their online friends. For access control, OverSoc employs point-to-point encryption and authentication, peers bootstrap private connections by exchanging the base of the PGP certificate and the profile overlays signature packet obtained in the "making friends" stage. Because the profile owner also is the CA, control of which could be distributed across the users resources, for all members of the overlay, they can easily revoke users from access to the profile overlay. [3] describes efficient mechanisms for overlay revocation through the use of broadcasting for immediate revocation and the use of DHT for indirect and permanent revocation.

The message board of a profile can be stored in two ways: distributed within the profile overlay via a data store or stored on the profile owner's personal computing devices. The distributed data store provides the profile when the owner is offline and also distributes the load for popular profiles. For higher availability, each peer always stores and provides all data in their profile when they are online. To ensure authenticity and integrity, peers sign their messages and each peer's certificate is available in the overlay as well as stored by mutual friends for verification. Messages that are unsigned are ignored by all members of the overlay. An ideal overlay for this purpose should support complex queries [18] allowing easy access to data stored chronologically, by content, by type, i.e., media, status updates, or message board discussions.

Private messaging in the profile overlay is unidirectional; only the profile owner can receive private messages using their overlay. To enforce this, a private message should be prepended with a symmetric key encrypted by the profile owners public key, the message should be appended by a signature of the message using the private key of the message sender, and the entire message encrypted by the symmetric key. This approach ensures that only the sender and the profile owner can decrypt the private message and verify the senders identity. The contents of the private message include the sender, time sent, and the subject. Messages are be stored in well known locations in the DHT, like "private messages for me", so that the profile owner can either poll the location.

\section{Active Peers}

The directory overlay should be used to assist in finding currently active peers in the profile overlays. By placing their node IDs at a well-known, unique per-profile overlay keys in the DHT, active peers can bootstrap incoming peers into the profile overlay. We implemented and evaluated this concept in [3]. Because the profile overlay members all use PKI to ensure membership, even if malicious peers insert their ID into the active list, it would be useless as the peer would only form connections with peers who also have a signed certificate. Extending from the earlier example, where Alice became Bob's friends, Figure 3 presents in detail how she would join his private overlay.

\section{E. Groups}

Groups can be considered extensions of profile overlays. The fundamental difference between a group and a profile is that a group lacks private messaging and has shared ownership. So just as a peer can find a profile in the directory by hashing the name of the user and other identifiable information, so can the user find the group. Like the certificate of the user, the members of a group sign the group's certificate to represent their membership to that group. In OverSoc, users request 
membership to the group like they do friendship requests, in response a group manager can sign their certificate allowing that member access to the group. Finally, the group can be bootstrapped in the same way as the profile overlay through the directory overlay.

The unique challenge presented by groups is the sharing of the CA task. A decentralized solution would be for all members of the group to be listed in the groups DHT and when a peer becomes a manager, they obtain a new signature packet that contains a user-defined component stating that they are managers. If an administrator loses their position, then all members who had their certificate signed by that administrator would need to obtain a new certificate. To avoid member churn, the owner could provide signature packets for all group members. Thus the managers just allow temporary access until the owner comes online and provides more permanent access.

\section{USER INTERACTION}

OverSoc consists of many components that are transparent to the user, the user experience should appear to the user no differently than an existing online social network. The OverSoc could be a downloadable application or a browser based Flash or Silverlight application. If the user, Bob, had already created an account, Bob would be presented with an interface showing their friends profiles. Based upon Bob's configuration, the social application could retrieve profile updates as he navigates to individual profiles or as soon as the application joins an individual profile overlay, reactive versus proactive profile querying.

If this was Bob's first time starting OverSoc, he would be presented with screens asking for his privacy preferences, such as whether or not he wants his information in the directory overlay, if he felt comfortable enough with the idea of people knowing he was a member of the social network and who his friends are. Then OverSoc would ask for personal information to populate his profile and to generate his directory information. At which point, the OverSoc would join the overlay and create Bob's private overlay. Bob could then start searching for friends, make friend requests, and respond to friend requests.

Recently, Bob had been thinking about his high school days and was curious if Alice was also a member of OverSoc, though Bob did not have Alice's e-mail address, just her first and last name. Bob enters Alice's name into the OverSoc search box and is presented by a list of Alice's. As Bob reviews each of the entries, he recognizes an Alice that is friend's with some of the same people Bob was in high school. Bob selects to become her friend. At which point, the OverSoc transparently inserts a friendship request to Alice and signs Alice's certificate so Alice can view Bob's profile. Of course that is because Bob has chosen to allow userinitiated friend requests access to his profile. Alice receives Bob's request, peruses his profile and feels fine becoming friends with Bob, which initiates a transparent process of signing Bob's certificate and placing the result in the public overlay. There is one problem though, when Bob receives Alice's signature and views her profile, he realizes that this is some other Alice. He quickly chooses to defriend her. This causes Bob's OverSoc instance to broadcast a revocation for Alice's signature and to store the revocation in the DHT. Alice, who was viewing Bob's profile, is notified of this sudden loss of trust and while she is able to view the contents of Bob's profile, which she has already accessed and obtained, she can no longer receive updates as members of Bob's overlay prevent her from accessing it.

In another instance, Bob bumped into Carol, who e-mailed Bob a copy of her certificate. Bob points OverSoc to the certificate, and OverSoc verifies that he wants to become friends with the identity associated with the certificate. When he accepts, OverSoc immediately submits a request to become Carol's friend. Carol receives notification and accepts Bob's friendship request. At this point, both Bob and Carol have transparently exchanged signed certificates and have mutual access to each other profiles. As Bob reads Carol's latest news, he remembers a funny personal story and that he would like to share with Carol. So he sends Carol a private message. Carol is offline though. The next time Carol goes online, her social application discovers the message and presents it to her. In this scenario, OverSoc has taken the private message, secured it with her public key and a symmetric key and signed it with his private key. After which, it inserts the message into the DHT and sends a notice to the event notification system, which detects that there were no listeners. When Carol's application comes online, it queries the DHT receiving the message. Prior to presenting Carol the message, the OverSoc decrypts and verifies the message.

The OverSoc architecture can leverage existing social networks to bootstrap trust. For example, consider Bob and David are two friends on Facebook. Bob joins a Facebook application called "OverSoc/Facebook Bridge", which stores a copy of his OverSoc certificate in his personal profile. Bob has been bragging to David about OverSoc and mentions to him how easy it is to migrate from Facebook to OverSoc using this application. So David joins OverSoc as well as the application. When David accesses the application, it pastes his certificate to his profile, notifies notifies him that he has a friend already using it, Bob, and that he can immediately sign Bob's certificate, and leaves a request for Bob to sign his certificate. Additionally, when David logs into OverSoc, he can leave a friend request there as well, so that the next time Bob accesses Facebook or OverSoc, he will receive David's request and can sign David's certificate. At which point, both will have access to each others OverSoc profile overlays.

\section{Challenges}

While structured P2P overlays have been well-studied in a variety of applications, their use in social profile overlays raises new interesting questions, including:

1) Handling small overlay networks - P2P overlay research typically focuses on networks larger than the typical user's friend count (Facebook's average is 130 ${ }^{1}$ ). Because social profile overlays are comparatively smaller, this can impact the reliability of the overlay and availability of profile data. A user can host their own profile; however when the

\footnotetext{
${ }^{1}$ http://www.facebook.com/press/info.php?statistics
} 
user is disconnected it is important that their profile remains available even under churn. It is thus important to characterize churn in this application to understand how to best approach this problem. An optional of per-user deployment of a virtual individual server (VIS) and the use of replication schemes aware of a user's resources provide possible directions to address this issue.

2) Overlay support for low throughput, unconnected devices - devices such as smart phones cannot constantly be actively connected to the overlay and the connection time necessary to retrieve something like a phone number may be too much to make this approach useful. Similar to the previous challenge, this approach could benefit from using a VIS enabling users access to their social overlays by proxy without establishing a direct connection to the overlay network.

3) Reliability of the directory and profile overlay Overlays are susceptible to attacks that can nullify their usefulness. While the profile overlay does have point-to-point security, in the public, directory overlay, the lack of any form centralization makes policing the system a complicated procedure. While our approach of appending friends list can assist users in making decisions on identity, it does not protect against denial of service attacks. For example, users could attempt create many similar identities in an attempt to overwhelm a user in their attempt to find a specific peer. Previous work has proposed methods to ensure the usability of overlays even while under attack. For the social overlay to be successful, we must identify which methods should be used. A possible approach is to replicate public information within a user's profile overlay thus providing an alternative directory overlay for querying prior to using the public directory overlay.

4) Social profile data storage - In previous works, DHTs have been used as the building blocks to form more complex distributed data stores as presented in Past [19] and Kosha [20]. Application of data stores will be heavily dependent on the churn rate associated with the overlay. If the system lacks any reasonably stable membership, large data files may be corrupted while smaller data sets are completely lost. Ideally, the usage model would be similar to those of Skype and Twitter, which have active processes for the duration of the computers usage. In an environment like this, data storage would be limited only by the available bandwidth of the participants.

\section{CONCLUSION}

In this paper, we proposed OverSoc, a system that constructs a decentralized online social network through the use of structure P2P overlays. P2P systems, in general, have very nice properties that make them attractive for average users. Namely, they require minimal configuration and organization of resources, in addition, P2P systems naturally handle increased demand due to additional peers. Our approach is based upon the use of a multiple overlay system, where all users join a public directory overlay which assists in finding friends and groups and bootstrapping into their private overlays. When making a friendship or joining groups, peers exchange signatures that enable access to each others overlays. Each private overlay employs point-to-point encryption and authentication so that only trusted peers can access the overlays. The use of unique overlays for each profile and group allows a clean abstraction for privacy purposes that simplifies security handling of access to private data. Existing work in the realm of structured overlays describes mechanisms for efficiently and securely storing profile information into the profile overlay. Our proposed system returns control of the social network and more importantly users' identity to the users and eliminates the need for centralized social networks. The most important insight in this paper is that structured overlays can be used to efficiently organize and maintain a social network.

\section{REFERENCES}

[1] J. C. Perez, "Facebook's beacon more intrusve than previously thought," http://www.pcworld.com/article/140182/facebooks_beacon_ more_intrusive_than_previously_thought.html, 2007.

[2] S. Buchegger and A. Datta, "A case for P2P infrastructure for social networks - opportunities \& challenges," in WONS '09: The Sixth International Conference on Wireless On-demand Network Systems and Services, 2009.

[3] D. I. Wolinsky, K. Lee, T. W. Choi, P. O. Boykin, and R. Figueiredo, "Virtual private overlays: Secure group communication in NATconstrained environments," January 2010.

[4] T. Klingberg and R. Manfredi, "Gnutella 0.6," http://rfc-gnutella. sourceforge.net/src/rfc-0_6-draft.html, June 2002.

[5] hex, "The fasttrack protocol," http://cvs.berlios.de/cgi-bin/viewcvs.cgi/ gift-fasttrack/giFT-FastTrack/PROTOCOL, September 2004.

[6] A. Rowstron and P. Druschel, "Pastry: Scalable, decentralized object location and routing for large-scale peer-to-peer systems," in IFIP/ACM International Conference on Distributed Systems Platforms (Middleware), November 2001.

[7] I. Stoica and et al., "Chord: A scalable Peer-To-Peer lookup service for internet applications," in SIGCOMM, 2001.

[8] G. S. Manku, M. Bawa, and P. Raghavan, "Symphony: distributed hashing in a small world," in USITS, 2003.

[9] P. Maymounkov and D. Mazières, "Kademlia: A peer-to-peer information system based on the XOR metric," in IPTPS '02, 2002.

[10] S. Ratnasamy, P. Francis, S. Shenker, and M. Handley, "A scalable content-addressable network," in In Proceedings of ACM SIGCOMM, 2001.

[11] V. Ramasubramanian and E. G. Sirer, "Beehive: O(1)lookup performance for power-law query distributions in peer-to-peer overlays," in Symposium on Networked Systems Design and Implementation, 2004.

[12] D. I. Wolinsky, P. S. Juste, P. O. Boykin, and R. Figueiredo, "Addressing the p2p bootstrap problem for small overlay networks," , 2010.

[13] S. Buchegger, D. Schiöberg, L. H. Vu, and A. Datta, "Peerson: P2p social networking: early experiences and insights," in SNS '09: Proceedings of the Second ACM EuroSys Workshop on Social Network Systems, 2009.

[14] A. Shakimov, H. Lim, L. P. Cox, and R. Caceres, "Vis-à-vis:online social networking via virtual individual servers," Tech. Rep., May 2008.

[15] L. A. Cutillo, R. Molva, and T. Strufe, "Privacy preserving social networking through decentralization," in Wireless On-Demand Network Systems and Services (WONS'09).

[16] S. M. A. Abbas, J. A. Pouwelse, D. H. J. Epema, and H. J. Sips, "A gossip-based distributed social networking system," in Enabling Technologies, IEEE International Workshops on, 2009.

[17] J. Albrecht, D. Oppenheimer, A. Vahdat, and D. A. Patterson, "Design and implementation trade-offs for wide-area resource discovery," in ACM Trans. Internet Technol., 2008.

[18] M. Harren, J. M. Hellerstein, R. Huebsch, B. T. Loo, S. Shenker, and I. Stoica, "Complex queries in dht-based peer-to-peer networks," in IPTPS '01: Revised Papers from the First International Workshop on Peer-to-Peer Systems, 2002.

[19] A. Rowstron and P. Druschel, "Storage management and caching in PAST, a large-scale, persistent peer-to-peer storage utility," in Symposium on Operating Systems Principles (SOSP'01).

[20] A. R. Butt, T. A. Johnson, Y. Zheng, and Y. C. Hu, "Kosha: A peer-to-peer enhancement for the network file system," in IEEE/ACM Supercomputing 2004. 